\title{
ANSICHTEN ANGEHENDER DAF-LEHRENDER ZUR DRAMAPÄDAGOGIK IM FREMDSPRACHENUNTERRICHT
}

Seit der Schaffung des Gemeinsamen Europäischen Referenzrahmens (weiter GeR) im Jahr 2001 ist das oberste Ziel des Fremdsprachenunterrichts Lernende zu befähigen in verschiedenen gesellschaftlichen Diskursen sprachlich adäquat zu handeln. Die Entwicklung der kommunikativen Kompetenz in der Fremdsprache wurde dadurch zum Postulat des Fremdsprachenunterrichts. Dieses Ziel sollte sich auch in methodisch-didaktischen Überlegungen bzw. Prinzipien bei der Planung des Fremdsprachenunterrichts widerspiegeln und bei dessen Durchführung realisieren. Eine der Möglichkeiten, den Fremdsprachenunterricht als Ort der Interaktion zwischen allen Beteiligten zu gestalten, bietet auch der Einsatz der Dramapädagogik.

Obwohl es scheint, dass dramapädagogische Elemente, die das ganzheitliche Lernen unterstützen, im Widerspruch zum pragmatisch ausgerichteten GeR stehen oder laut Schmenk (2004: 15) völlig aus dem GeR ausgeschlossen werden, berichten z. B. Neumayer (2002), Bünger (2010) und Birnbaum (2013) von positiven Effekten dramapädagogischer Elemente auf das Fremdsprachenlernen - und zwar sowohl in schulischen wie auch in universitären Kontexten. Viele erfolgreiche praktische Beispiele und theoretische Überlegungen sind auch in Tagungsbänden von Dramapädagogik-Tagen zu finden (zugänglich auf der Webseite https://dramapaedagogik.de/de/). Auch Haack (2018) zeigte in seiner umfangreichen Studie, dass dramapädagogische Elemente in der Lehrerausbildung einen positiven Beitrag zur persönlichen und professionellen Kompetenzerweiterung der Lehrenden leisten könnten.

Da der Einsatz von neuen oder weniger bekannten Lehr-Lernkonzepten im Unterricht jedoch stark davon abhängig ist, wie Lehrende diese Konzepte bewerten und welche Erfahrungen sie selbst damit haben/gemacht haben, ist es von Bedeutung, dass Lehrende sowohl in der Ausbildungszeit wie auch bei Fortbildungen mit unterschiedlichen LehrLernmethoden vertraut gemacht werden und sie möglichst viele Methoden auch praktisch ausprobieren, damit sie über ein breites Methodenrepertoire verfügen und es später in ihren eigenen Unterricht miteinbeziehen. Im vorliegenden Artikel wird auf das Konzept der 
Dramapädagogik für den Fremdsprachenunterricht näher eingegangen; darüber hinaus werden die Resultate der Befragung von angehenden DaF-Lehrenden präsentiert und ein dramapädagogischer Workshop als eine der Möglichkeiten der Verzahnung von Theorie und Praxis bewertet.

\section{DRAMAPÄDAGOGIK ALS KONZEPT DES FREMDSPRACHENLERNENS}

Dramapädagogik und Theaterpädagogik gehen in der Alltagssprache oft Hand in Hand. In der Fremdsprachendidaktik wird folgende Distinktion zwischen den beiden Konzepten (vgl. Sambanis 2016) gemacht:

\begin{tabular}{|c|c|}
\hline Dramapädagogik & Theaterpädagogik \\
\hline $\begin{array}{l}\text { - Prozessorientierung (Aufführung nicht } \\
\text { obligatorisch); } \\
\text { - Richtet sich an die Teilnehmer; } \\
\text { - Ziel: Dynamisches, mehrkanaliges Lernen } \\
\text { durch Imagination, Interpretation, } \\
\text { Dramatisierungsversuche, Probehandeln, } \\
\text { Feedback und Reflexion; } \\
\text { - Geeignet für kürzere Unterrichtssequenzen; }\end{array}$ & $\begin{array}{l}\text { - Produktorientierung (Aufführung } \\
\text { obligatorisch); } \\
\text { - Richtet sich an das Publikum; } \\
\text { - Ziel: Aufführung mit Vor- und } \\
\text { Nachbereitung; } \\
\text { - Vorgehen: Textauswahl, Rollenverteilung, } \\
\text { Techniker, Organisatorisches, Aufführung; } \\
\text { - Geeignet für komplexe und längere Arbeit } \\
\text { mit Texten z. B. in einer Theater-AG; }\end{array}$ \\
\hline
\end{tabular}

Abbildung 1: Unterschied zwischen Dramapädagogik und Theaterpädagogik (vgl. Sambanis 2016)

Dramapädagogik, wie in der Abbildung 1 stichwortartig präsentiert, zielt auf die Konstruktion neuen Wissens durch mehrkanaliges Lernen, indem Lernende mithilfe von Sprache und Körper eine Inszenierung vorbereiten, von der Lehrperson und anderen Beteiligten ein Feedback bekommen und zur eigenen Reflexion aufgefordert werden. Das neue sprachliche und nichtsprachliche Wissen entsteht in der "Sprachnotsituation“ (Tselikas 1999) und durch Selbstreflexion. Im Fokus stehen der Lernprozess und die Teilnehmer. Das Hauptziel ist nicht die Vermittlung ästhetischer Prinzipien, sondern die dramapädagogische Herangehensweise verfolgt stets pädagogische Ziele. Die Theaterpädagogik dagegen hat eine Theateraufführung zum Ziel, vermittelt ästhetische Komponenten und möchte eine Wirkung auf das Publikum ausüben. Dem Lernprozess wird eine kleinere Rolle zugewiesen. Vor Augen hat man stets das Publikum und das Endprodukt, also eine Theateraufführung.

Der Begriff Dramapädagogik wurde im deutschsprachigen Raum von Manfred Schewe in zahlreichen Beiträgen in fachdidaktische Diskussionen eingebracht. In seinem bekanntesten Werk aus dem Jahr 1993 ergänzte er die berühmte Maxime Pestalozzis und 
plädierte „für ein Lehren und Lernen mit Kopf, Herz, Hand und Fuß“ (Schewe 1993: 51). Er ging davon aus, dass verschiedene Fertigkeitsbereiche mithilfe von dramapädagogischen Elementen erfolgreich entwickelt werden könnten. In seinem ganzheitlichen Lehr-Lernkonzept der Dramapädagogik, das in Anlehnung an das britische „Drama in Education" entwickelt wurde, sollten im dramapädagogischen Unterricht situative, fiktive Kontexte geschaffen werden, [...] ,in denen Lehrende und Lernende sprachlich und nichtsprachlich in intensiver Weise handeln - die fremde Sprache wird „inszeniert“ (ebd.: 5). Schewe sieht den dramapädagogischen Fremdsprachenunterricht als kommunikativen Fremdsprachenunterricht, in dem ,[i]m Vordergrund [...] weniger die Einübung linguistisch-formaler Bestände [steht] (obwohl diese begleitend/integriert auch erfolgt!), sondern eine Konzentration auf die Erarbeitung/Mitteilung von Inhalten“ (ebd.: 181).

Im Vordergrund stehen aber nicht nur sprachliche Phänomene, sondern auch Versuche zur Schaffung einer Lehr-Lerngelegenheit, in der Lernende dazu aufgefordert werden, ihre Gedanken situationsgemäß zu versprachlichen. Dies geschieht in einer Als-obSituation, in der sich die Lernenden in eine andere Rolle hineinversetzen, spontan handeln, kreativ mit der Sprache umgehen und ihr sprachliches Wissen und Können selbst und mit anderen Lernenden konstruieren, indem sie realitätsnahe Probleme lösen usw. Dieser handlungs- und erlebnisorientierte Ansatz ermöglicht das Erlernen einer Fremdsprache mit allen Sinnen, denn Lernende werden aufgefordert sprachlich und körperlich in einem gelenkten oder improvisierten Unterrichtsetting zu handeln und über die sprachlichen und nicht-sprachlichen Phänomene zu reflektieren.

Der dramapädagogische Unterricht hat nicht zum Ziel schauspielerische Talente aufzudecken, sie zu entwickeln oder eine perfekte Theatervorstellung auf die Bühne zu bringen, sondern das kognitive, soziale, fertigkeitsorientierte und ästhetische Lernen mithilfe von verschiedenen Inszenierungstechniken zu fördern, wobei Lehrende und Lernende zu „Konstrukteure[n] von Handlungssituationen“ (ebd.: 408) werden, in denen ,[...] die Beteiligten nicht anders können als (körperlich) zu handeln. Und aus der Handlung heraus formiert sich die (fremde) Sprache (ebd.: 408).“

Auch Even (2003) behauptet, dass die Dramapädagogik eine ganzheitliche Vernetzung von handlungsorientierten, erfahrungsbezogenen, interaktiven und alternativen Ansätzen darstellt und ,[...] durch die bewusste Einbeziehung kinästhetischer Lernmomente bereits einen performativen Charakter in sich trägt" (Even 2014). Ähnlich auch Tselikas (1999: 21), die Dramapädagogik als einen Ansatz definiert, der „,die Mittel des Theaters zu pädagogischen Zwecken einsetzt“. „Im Vordergrund steht dabei nicht primär das Ergebnis, nämlich die Produktion eines Theaterstücks, sondern der Lernprozess in allen seinen Dimensionen: physisch, ästhetisch (sinnlich), emotional und kognitiv" (ebd.).

Das Erlernen einer Fremdsprache mit dem dramapädagogischen Ansatz vergleicht Tselikas (ebd.) mit dem für das Theater charakteristischen Prinzip des Rituals und des Risikos. Lernende befinden sich beim sprachlichen Handeln stets in einer Situation zwischen Spontanität und Kreativität - zwischen dem Risiko und dem Befolgen fremdsprachlicher 
Regeln, Konventionen - Ritualen. Dabei sollen sie das Risiko eingehen und auf die Sprache und körperliche Signale zurückgreifen, um ihre Gedanken auszudrücken.

Dem dramapädagogischen Ansatz sind zwei Prinzipien inhärent, und zwar Empathie und Reflexion (vgl. Bonnet und Küppers 2011). Lernende werden aufgefordert, „die reale Schulwirklichkeit zu verlassen und sich auf eine fiktive Wirklichkeit einzulassen" (ebd.: 41).

Durch den Einsatz dramapädagogischer Elemente werden laut Tselikas (1999: 42) „Sprachnotsituationen“ geschaffen, in denen Lernende eine starke Sprechabsicht und Notwendigkeit des sprachlichen und körperlichen Ausdrucks zeigen; dies resultiert nicht nur in der situativen Kommunikation zwischen den Beteiligten, sondern auch in neurologischen Prozessen im Gehirn. Auch laut Sambanis $(2013,2016)$ tragen körperliche Bewegungen dazu bei, die Fremdsprache im Gehirn zu verankern und unterstützen das langfristige Behalten des Gelernten.

Die Rolle der Körpersprache beim Sprachenlernen wurde lange nicht beachtet oder als relevanter Faktor angesehen, jedoch zeigen aktuelle Studien aus dem Bereich der Neurowissenschaft und Neurodidaktik gerade das Gegenteil. Das Aufnehmen, Verarbeiten und Abspeichern von Lerninhalten wird durch die von den Lernenden ausgeführten Bewegungen sehr unterstützt (vgl. Sambanis 2016).

Um die Ansichten der Studierenden zur Effizienz der Dramapädagogik auf das Fremdsprachenlernen zu erkunden, wurde ein zweiwöchiger Workshop (11 Unterrichtsstunden) organisiert. Zu Beginn fand eine Gesprächsrunde über bisherige Erfahrungen der Studierenden statt (ungefähr 30 Minuten), im Anschluss daran folgten eine Vorlesung zu den theoretischen Hintergründen der Dramapädagogik sowie eine Präsentation praktischer Beispiele anhand von kurzen Videos (2 Stunden). Darauffolgend wurden verschiedene dramapädagogische Techniken (heißer Stuhl, Gedankenallee, Stimmenskulptur, Standbilder, Rollenspiele, Dialoge vorspielen ...) kurz präsentiert und von den Studierenden erprobt und reflektiert (8 Stunden). Als Impulse für Inszenierungen dienten Prosatexte, Gedichte, Texte aus Kursbüchern und Sachtexte aus verschiedenen Schulfächern. Am Ende des Workshops wurde eine schriftliche Befragung durchgeführt (30 Minuten).

\subsection{Forschungsfrage}

Das Ziel der Untersuchung war eine Antwort auf folgende Frage zu finden:

1) Wie bewerten angehende DaF-Lehrende die Effizienz der Dramapädagogik für das Fremdsprachenlernen? 


\subsection{Methode}

Um die erwähnte Forschungsfrage zu beantworten, wurde eine deskriptiv-kausale, nichtexperimentelle Methode ausgewählt. Zur Datenerhebung bedienten wir uns einer schriftlichen Befragung. Es wurde ein Fragebogen mit sieben geschlossenen Fragen entwickelt. Der Fragebogen besteht aus zwei Teilen: Der erste Teil mit 6 Fragen bezieht sich auf die Einstellung der Probanden zum Theater. Der zweite Teil umfasst zwei weitere Fragen. Die sechste Frage enthält 40 Aussagen - Items zur Dramapädagogik, die auf einer 5-stufigen Likert-Skala abgefragt wurden (1-stimmt gar nicht - 5 stimmt völlig).

\subsection{Forschungsteilnehmer}

An der Umfrage nahmen 41 DaF-Studierende teil, die im Studienjahr 2019/2020 ihr pädagogisches Masterstudium absolvierten und im Rahmen des Studiums die Veranstaltungen Pädagogisches Praktikum I und II besuchten. Unsere Voraussetzung war: Die angehenden DaF-Lehrenden sollten selbst gewisse Lehrmethoden ausprobieren, um diese später in ihrem Unterricht in verschiedenen schulischen Kontexten einzusetzen. Diesbezüglich wurde ein Teil der Veranstaltungen als Workshop organisiert, in dem die angehenden DaF-Lehrenden einige Grundprinzipien der Dramapädagogik kennenlernten und einige Methoden selbst ausprobierten. Am Ende des Workshops wurden die Daten mithilfe des Fragebogens erhoben.

\subsection{Datenanalyse}

Die eingesammelten Fragebögen wurden zuerst ins Computerprogramm Excel übertragen und danach ausgewertet. In die Analyse wurden 34 vollständig ausgefüllte Fragebögen einbezogen $(\mathrm{N}=34)$. Berechnet wurden die Häufigkeit der Antworten (f, $\mathrm{f} \%$ ), der Mittelwert $(\bar{x})$ und die Standardabweichung $(\sigma)$. Die Daten werden tabellarisch mithilfe der deskriptiven Statistik dargestellt.

\subsection{Resultate und Interpretation}

Das Ziel des ersten Teils des Fragebogens war, sich ein Bild von der Einstellung der Probanden zum Theater und ihren eigenen Erfahrungen damit zu machen. Das Ziel des zweiten Teils war die Bewertung der dramapädagogischen Methode nach dem Workshop.

Aus der Tabelle 1 ist zu entnehmen, dass die Mehrheit (65\%) der Teilnehmer keine eigenen Erfahrungen mit einer Theater-AG hat und auch keinen Wunsch hat in einem Studententheater mitzuwirken (53\%). Jedoch gibt eine große Mehrheit (91\%) an, dass sie eine Vorliebe für das Theater hat. Die Daten bestätigen unsere Erwartungen, denn Dramapädagogik und Theaterpädagogik sind in slowenischen Mittelschulen bis auf wenige Ausnahmen nicht sehr verbreitet. Auch gibt es an nahezu keiner Schule mehr ein deutschsprachiges Theater. Zum einen mangelt es an Fortbildungen zum Thema Dramapädagogik und zum 
anderen erlebte das Fach Deutsch als Fremdsprache in den letzten Jahren einige Veränderungen, die nicht zugunsten des Faches waren (siehe Kosevski Puljić, Retelj, 2019), was sicherlich auch in diesem Bereich Spuren hinterlassen hat.

Tabelle 1: Einstellung zum Theater und Erfahrungen damit

\begin{tabular}{|l|c|c|c|}
\hline \multicolumn{1}{|c|}{ Fragen } & Ja (f, f\%) & Nein (f, f\%) & N \\
\hline 1. Sind/Waren Sie Mitglied einer Theater-AG? & 12 & 22 & 34 \\
& $35 \%$ & $65 \%$ & $100 \%$ \\
\hline $\begin{array}{l}\text { 2. Würden Sie in einer Studententheater-AG } \\
\text { an der Abteilung für Germanistik mitwirken, } \\
\text { wenn es eine gäbe? }\end{array}$ & 16 & 18 & 34 \\
\hline 3. Mögen Sie Theater? & 31 & $53 \%$ & $100 \%$ \\
& $91 \%$ & 3 & 34 \\
\end{tabular}

Um herauszufinden, ob Studierende auch selbst ins Theater gehen und am Theaterleben teilnehmen, wollten wir wissen, wie oft sie sich dorthin begeben und sich eine Theateraufführung ansehen (Frage 4). Die Resultate zeigen, dass nur eine befragte Person nie ins Theater geht, die Mehrheit (21 Studierende) sich selten ein Theaterstück ansehen, 10 Studierende manchmal ins Theater gehen; die Antworten „oft“" und „,sehr oft“ hat je eine befragte Person ausgewählt. Eigentlich sind die Zahlen ziemlich überraschend, denn wie in der Tabelle 1 zu sehen ist, sagen 91 \% der Studierenden, dass sie Theater mögen. Als Studierende haben sie auch ermäßigte Theaterkarten und werden an der Abteilung ständig über das Theaterprogramm informiert. Zugleich weisen diese Resultate auch darauf hin, dass eine Vorliebe für das Theater z. B. noch nicht bedeutet, dass man auf diesem Gebiet auch selbst aktiv wird. Entsprechende Parallelen können wir auch zu den Unterrichtsmethoden ziehen: Wenn Lehrende keine oder wenige eigene Erfahrungen mit einer gewissen Methode haben, bestehen nur geringe Chancen, dass sie sie auch in ihren Unterricht miteinbeziehen.

Tabelle 2: Frage 4: Wie oft gehen Sie ins Theater?

\begin{tabular}{|c|c|c|c|c|c|}
\hline \multicolumn{7}{|c|}{ Frage 4: Wie oft gehen Sie ins Theater? } \\
\cline { 1 - 4 } 1 (nie) & 2 & 3 & 4 & 5 (sehr oft) & \multirow{N}{*}{} \\
\cline { 1 - 3 } & Grad der Zustimmung (f, f\%) & \multirow{2}{*}{34} \\
31 & 21 & 10 & 1 & 1 & $100 \%$ \\
\hline
\end{tabular}

Da Dramapädagogik in Slowenien nicht zu den Mainstream-Methoden gehört und nur von wenigen DaF-Lehrkräften praktiziert wird, wollten wir wissen, ob die Befragten schon einige Elemente der Dramapädagogik in der Grund- oder Mittelschule im 
Unterricht kennengelernt haben. Die Resultate sind in der Tabelle 3 dargestellt. Die Hälfte der Teilnehmer hatte nur selten Fremdsprachenunterricht mit dramapädagogischen Elementen, 11 Studierende manchmal, oft und sehr oft nur 3 und genauso 3 hatten noch nie Fremdsprachenunterricht mit Dramapädagogik.

Tabelle 3: Frage 5: Wie oft hatten Sie Fremdsprachenunterricht mit dramapädagogischen Elementen?

\begin{tabular}{|c|c|c|c|c|c|}
\hline \multicolumn{7}{|c|}{ Frage 5: Wie oft hatten Sie bis jetzt Fremdsprachenunterricht } \\
mit Elementen der Dramapädagogik ?
\end{tabular}

Die sechste Frage beinhaltet 40 Aussagen. Sie werden folgenden Kategorien zugeordnet: Wortschatzlernen, Grammatik, Entwicklung von fremdsprachlicher Kompetenz/ Sprachfertigkeit, Entwicklung literarischer Kompetenzen, Emotionen, Lernprozess und Lernformen und Entwicklung diverser Kompetenzen. Die Studierenden bewerteten den Grad der Zustimmung auf einer fünfstufigen Likert-Skala von 1 (Ich stimme überhaupt nicht zu.) bis 5 (Ich stimme völlig zu.). Die Effizienz der einzelnen Kategorien wurde mithilfe der Mittelwerte auf folgende Weise definiert: 5,00-4,50 - sehr hohe Effizienz; 4,49-4,00 hohe Effizienz; 3,99-3,00 mittelmäßige Effizienz; 2,99-2,00 geringe Effizienz; $1,99-1.00$ keine Effizienz.

\subsubsection{Kategorie Vokabellernen}

Die Teilnehmenden sehen dramapädagogische Elemente im Bereich Vokabellernen sowohl für das Erlernen von neuen Vokabeln $(\overline{\mathrm{x}}=4,56)$ wie auch für das langfristige Behalten der Wörter $(\overline{\mathrm{x}}=4,53)$ als wichtigen Faktor an. Die Mehrheit denkt auch, dass der richtige Gebrauch von festen Phrasen mit dramapädagogischen Elementen gefördert wird $(\overline{\mathrm{x}}=3,59)$. Da sich die zukünftigen DaF-Lehrenden bei den Inszenierungen im Workshop stets in Situationen befanden, in denen sie sich sprachlich angemessen ausdrücken mussten und ihre Gedanken und Gefühle überzeugend vermitteln wollten, gehen wir davon aus, dass sie selbst einsahen, über welche sprachlichen Mittel sie verfügen und welche ihnen noch fehlen. Dabei bedienten sie sich des Wörterbuches, fragten andere Beteiligte und benutzten die Phrasen mehrmals in sinnvollen Kontexten. In diese Kategorie wurden zwei weitere Aussagen eingeordnet, und zwar die Behandlung von landeskundlichen und Tabu-Themen, denn beide Themen sind eng mit dem Lernen der unbekannten Vokabeln verbunden. Auch hier scheint Dramapädagogik von Bedeutung zu sein. Der Mittelwert für die Kategorie Wortschatzlernen beträgt $\bar{x}=4,00$ und Dramapädagogik erweist sich damit als Methode mit einer hohen Effizienz. 
Tabelle 4: Wortschatzlernen

\begin{tabular}{|c|c|c|c|c|c|c|c|c|}
\hline \multirow[t]{2}{*}{ Frage 6 - Aussagen } & 1 & 2 & 3 & 4 & 5 & \multirow[t]{2}{*}{$\mathbf{N}$} & \multirow[t]{2}{*}{$(x)$} & \multirow{2}{*}{$\begin{array}{l}(\sigma) \\
\text { Std. }\end{array}$} \\
\hline & \multicolumn{5}{|c|}{ Grad der Zustimmung (f, f\%) } & & & \\
\hline $\begin{array}{l}\text { 1. DP im FU fördert das Erlernen } \\
\text { von neuen Vokabeln. }\end{array}$ & $\begin{array}{c}0 \\
0 \%\end{array}$ & $\begin{array}{c}0 \\
0 \%\end{array}$ & $\begin{array}{l}0 \\
0\end{array}$ & $\begin{array}{c}15 \\
44 \%\end{array}$ & $\begin{array}{c}19 \\
56 \%\end{array}$ & $\begin{array}{c}34 \\
100 \%\end{array}$ & 4,56 & 0,50 \\
\hline $\begin{array}{l}\text { 2. Mit DP im FU merken sich } \\
\text { Lernende Wortschatz } \\
\text { langfristiger als sonst. }\end{array}$ & $\begin{array}{c}0 \\
0 \%\end{array}$ & $\begin{array}{c}0 \\
0 \%\end{array}$ & $\begin{array}{c}0 \\
0 \%\end{array}$ & $\begin{array}{c}16 \\
47 \%\end{array}$ & $\begin{array}{c}18 \\
53 \%\end{array}$ & $\begin{array}{c}34 \\
100 \%\end{array}$ & 4,53 & 0,50 \\
\hline $\begin{array}{l}\text { 3. DP im FU macht Lernende auf } \\
\text { den richtigen Gebrauch von } \\
\text { Phrasen (Chunks) aufmerksam. }\end{array}$ & $\begin{array}{c}2 \\
6 \%\end{array}$ & $\begin{array}{c}2 \\
6 \%\end{array}$ & $\begin{array}{c}12 \\
35 \%\end{array}$ & $\begin{array}{c}10 \\
29 \%\end{array}$ & $\begin{array}{c}8 \\
24 \%\end{array}$ & $\begin{array}{c}34 \\
100 \%\end{array}$ & 3,59 & 1,09 \\
\hline $\begin{array}{l}\text { 4. Mit DP kann Landeskunde } \\
\text { besonders gut vermittelt } \\
\text { werden. }\end{array}$ & $\begin{array}{c}1 \\
3 \%\end{array}$ & $\begin{array}{c}4 \\
12 \%\end{array}$ & $\begin{array}{c}10 \\
29 \%\end{array}$ & $\begin{array}{c}10 \\
29 \%\end{array}$ & $\begin{array}{c}9 \\
27 \%\end{array}$ & $\begin{array}{c}34 \\
100 \%\end{array}$ & 3,59 & 1,22 \\
\hline $\begin{array}{l}\text { 5. DP ermöglicht die Bearbeitung } \\
\text { von Tabu-Themen im FU. }\end{array}$ & $\begin{array}{c}0 \\
0 \%\end{array}$ & $\begin{array}{c}2 \\
6 \%\end{array}$ & $\begin{array}{c}12 \\
35 \%\end{array}$ & $\begin{array}{c}12 \\
35 \%\end{array}$ & $\begin{array}{c}8 \\
24 \%\end{array}$ & $\begin{array}{c}34 \\
100 \%\end{array}$ & 3,76 & 0,88 \\
\hline
\end{tabular}

\subsubsection{Kategorie Grammatik}

Wie in der Tabelle $5 \mathrm{zu}$ sehen ist, werden dramapädagogische Elemente auch für das Erlernen grammatischer Strukturen in der Fremdsprache als nützliche Lernmethode bewertet. Den positivsten Einfluss sehen Studierende in der Förderung der Sprachrichtigkeit $(\overline{\mathrm{x}}=4,00)$ und in der Sensibilisierung für sprachliche Phänomene $(\overline{\mathrm{x}}=3,71)$. Da die Teilnehmer in ihrer Schulzeit nur wenige Erfahrungen mit Dramapädagogik gesammelt hatten, waren wir eher positiv überrascht, dass so viele an die Effizienz dramapädagogischer Elemente beim Lernen grammatischer Strukturen glauben $(\bar{x}=3,53)$. Wir vermuten, dass das Selbsterproben in diesem Fall eine wichtige Rolle spielte. Der Mittelwert für die Kategorie Grammatik beträgt 3,75, was als durchschnittliche Effizienz bestimmt wurde.

\section{Tabelle 5: Grammatik}

\begin{tabular}{|c|c|c|c|c|c|c|c|c|}
\hline \multirow[t]{2}{*}{ Frage 6 - Aussagen } & 1 & 2 & 3 & 4 & 5 & \multirow[t]{2}{*}{$\mathbf{N}$} & \multirow[t]{2}{*}{$(x)$} & \multirow{2}{*}{$\begin{array}{l}(\sigma) \\
\text { Std }\end{array}$} \\
\hline & \multicolumn{5}{|c|}{ Grad der Zustimmung (f, f\%) } & & & \\
\hline $\begin{array}{l}\text { 6. DP sensibilisiert Lernende für sprachli- } \\
\text { che Phänomene der Zielsprache. }\end{array}$ & $\begin{array}{c}1 \\
3 \%\end{array}$ & $\begin{array}{c}3 \\
9 \%\end{array}$ & $\begin{array}{c}11 \\
32 \%\end{array}$ & $\begin{array}{c}9 \\
27 \%\end{array}$ & $\begin{array}{c}10 \\
29 \%\end{array}$ & $\begin{array}{c}34 \\
100 \%\end{array}$ & 3,71 & 1,07 \\
\hline $\begin{array}{l}\text { 7. Mit DP werden neue grammatische } \\
\text { Strukturen schneller als beim } \\
\text { traditionellen FU gelernt. }\end{array}$ & $\begin{array}{c}3 \\
9 \%\end{array}$ & $\begin{array}{c}2 \\
6 \%\end{array}$ & $\begin{array}{c}11 \\
32 \%\end{array}$ & $\begin{array}{c}10 \\
29 \%\end{array}$ & $\begin{array}{c}8 \\
24 \%\end{array}$ & $\begin{array}{c}34 \\
100 \%\end{array}$ & 3,53 & 1,17 \\
\hline 8. DP fördert Sprachrichtigkeit. & $\begin{array}{c}0 \\
0 \%\end{array}$ & $\begin{array}{c}0 \\
0 \%\end{array}$ & $\begin{array}{c}7 \\
20 \%\end{array}$ & $\begin{array}{c}20 \\
60 \%\end{array}$ & $\begin{array}{c}7 \\
20 \%\end{array}$ & $\begin{array}{c}34 \\
100 \%\end{array}$ & 4,00 & 0,64 \\
\hline
\end{tabular}




\subsubsection{Kategorie Entwicklung von fremdsprachlicher Kompetenz/ Sprachfertigkeit}

Die Kategorie Entwicklung von fremdsprachlicher Kompetenz/Sprachfertigkeiten umfasst Aussagen, die sich auf die traditionellen vier Fertigkeiten (Lesen, Schreiben, Sprechen, Hören) beziehen. Bei allen Fertigkeiten sind die Mittelwerte ( $\overline{\mathrm{x}})$ der Zustimmung sehr hoch (von 4,03 bis und 4,47), das heißt, Dramapädagogik wird als effiziente Lernmethode für die Entwicklung dieser Fertigkeiten im Fremdsprachenunterricht angesehen. Die einzige Fertigkeit, die laut den Studierenden besser mit anderen Methoden gefördert werden kann, ist das Schreiben $(\bar{x}=2,88)$. Dramapädagogik ist eine für Teilnehmende sehr effiziente Methode für die Kategorie Entwicklung von fremdsprachlicher Kompetenz. Der Mittelwert beträgt 4,06.

Tabelle 6: Sprachfertigkeiten

\begin{tabular}{|c|c|c|c|c|c|c|c|c|}
\hline \multirow[t]{2}{*}{ Frage 6 - Aussagen } & 1 & 2 & 3 & 4 & 5 & \multirow[t]{2}{*}{$\mathbf{N}$} & \multirow[t]{2}{*}{$(\mathbf{x})$} & \multirow{2}{*}{$\begin{array}{l}(\sigma) \\
\text { Std. }\end{array}$} \\
\hline & \multicolumn{5}{|c|}{ Grad der Zustimmung (f, f\%) } & & & \\
\hline $\begin{array}{l}\text { 9. Mit DP können alle vier Fertigkeiten } \\
\text { (Schreiben, Sprechen, Lesen, Hören) } \\
\text { gefördert werden. }\end{array}$ & $\begin{array}{c}0 \\
0 \%\end{array}$ & $\begin{array}{c}3 \\
9 \%\end{array}$ & $\begin{array}{c}4 \\
12 \%\end{array}$ & $\begin{array}{c}13 \\
38 \%\end{array}$ & $\begin{array}{c}14 \\
41 \%\end{array}$ & $\begin{array}{c}34 \\
100 \%\end{array}$ & 4,12 & 0,93 \\
\hline $\begin{array}{l}\text { 10. DP hilft Lernenden ihre } \\
\text { kommunikative Kompetenz zu } \\
\text { entwickeln. }\end{array}$ & $\begin{array}{c}0 \\
0 \%\end{array}$ & $\begin{array}{c}0 \\
0 \%\end{array}$ & $\begin{array}{c}3 \\
9 \%\end{array}$ & $\begin{array}{c}12 \\
35 \%\end{array}$ & $\begin{array}{c}19 \\
56 \%\end{array}$ & $\begin{array}{c}34 \\
100 \%\end{array}$ & 4,47 & 0,65 \\
\hline $\begin{array}{l}\text { 11. DP im FU wirkt sich positiv auf die } \\
\text { Aussprache aus. }\end{array}$ & $\begin{array}{c}0 \\
0 \%\end{array}$ & $\begin{array}{c}1 \\
3 \%\end{array}$ & $\begin{array}{c}7 \\
20 \%\end{array}$ & $\begin{array}{c}7 \\
20 \%\end{array}$ & $\begin{array}{c}19 \\
56 \%\end{array}$ & $\begin{array}{c}34 \\
100 \%\end{array}$ & 4,29 & 0,89 \\
\hline $\begin{array}{l}\text { 12. DP im FU fördert die } \\
\text { Sprachflüssigkeit. }\end{array}$ & $\begin{array}{c}0 \\
0 \%\end{array}$ & $\begin{array}{c}0 \\
0 \%\end{array}$ & $\begin{array}{c}7 \\
20 \%\end{array}$ & $\begin{array}{c}11 \\
32 \%\end{array}$ & $\begin{array}{c}16 \\
47 \%\end{array}$ & $\begin{array}{c}34 \\
100 \%\end{array}$ & 4,26 & 0,78 \\
\hline 13. DP im FU fördert die Interaktion. & $\begin{array}{c}0 \\
0 \%\end{array}$ & $\begin{array}{c}0 \\
0 \%\end{array}$ & $\begin{array}{c}7 \\
20 \%\end{array}$ & $\begin{array}{c}8 \\
24 \%\end{array}$ & $\begin{array}{c}19 \\
56 \%\end{array}$ & $\begin{array}{c}34 \\
100 \%\end{array}$ & 4,35 & 0,80 \\
\hline 14. DP fördert die Lesekompetenz. & $\begin{array}{c}0 \\
0 \% \\
0\end{array}$ & $\begin{array}{c}1 \\
3 \%\end{array}$ & $\begin{array}{c}8 \\
24 \%\end{array}$ & $\begin{array}{c}12 \\
35 \%\end{array}$ & $\begin{array}{c}13 \\
38 \%\end{array}$ & $\begin{array}{c}34 \\
100 \% \\
\end{array}$ & 4,09 & 0,85 \\
\hline $\begin{array}{l}\text { 15. DP im FU fördert die Entwicklung der } \\
\text { Hörkompetenz in der Fremdsprache. }\end{array}$ & $\begin{array}{c}1 \\
3 \%\end{array}$ & $\begin{array}{c}1 \\
3 \%\end{array}$ & $\begin{array}{c}4 \\
12 \%\end{array}$ & $\begin{array}{c}18 \\
53 \%\end{array}$ & $\begin{array}{c}10 \\
29 \%\end{array}$ & $\begin{array}{c}34 \\
100 \%\end{array}$ & 4,03 & 0,89 \\
\hline $\begin{array}{l}\text { 16. DP im FU hat einen positiven } \\
\text { Effekt auf die Entwicklung der } \\
\text { Schreibkompetenz. }\end{array}$ & $\begin{array}{c}2 \\
6 \%\end{array}$ & $\begin{array}{c}9 \\
27 \%\end{array}$ & $\begin{array}{c}15 \\
44 \%\end{array}$ & $\begin{array}{c}7 \\
20 \%\end{array}$ & $\begin{array}{c}1 \\
3 \%\end{array}$ & $\begin{array}{c}34 \\
100 \%\end{array}$ & 2,88 & 0,90 \\
\hline
\end{tabular}

\subsubsection{Kategorie Lernprozess und Lernformen}

Die Kategorie Lernprozess und Lernformen umfassen Aussagen, die sich auf die Lernmethoden, Arbeits- und Sozialformen beziehen. Auch in dieser Hinsicht bewerteten die Teilnehmer Dramapädagogik als effektive Unterrichtsmethode. Die größten Vorteile der Dramapädagogik sehen die Studierenden im Lernen auf eine kommunikative Weise 
$(\overline{\mathrm{x}}=4,47)$, im kooperativen und aktiven Lernen, das allen Beteiligten, unabhängig von ihrem Sprachniveau, eine Teilnahme am Unterricht ermöglicht $(\bar{x}=4,15)$. Obwohl die Individualität der Lernenden $(\overline{\mathrm{x}}=4,12)$ und der authentische Sprachgebrauch $(\overline{\mathrm{x}}=3,82)$ durch Dramapädagogik stark gefördert werden, beurteilt ein großer Teil der Studierenden diese Methode als Mittel zur Belebung des Unterrichts $(\overline{\mathrm{x}}=3,18)$ und nicht als wichtigste LehrLernmethode. Der Mittelwert für diese Kategorie beträgt 3,94; der Dramapädagogik wird hiermit eine durchschnittliche bzw. mittelmäßige Effizienz zugeteilt.

Tabelle 7: Lernprozess und Lernformen

\begin{tabular}{|c|c|c|c|c|c|c|c|c|}
\hline \multirow[t]{2}{*}{ Frage 6 - Aussagen } & 1 & 2 & 3 & 4 & 5 & \multirow[t]{2}{*}{$\mathbf{N}$} & \multirow[t]{2}{*}{$(\mathbf{x})$} & \multirow{2}{*}{$\begin{array}{l}(\sigma) \\
\text { Std. }\end{array}$} \\
\hline & \multicolumn{5}{|c|}{ Grad der Zustimmung (f, f\%) } & & & \\
\hline $\begin{array}{l}\text { 17. DP ermöglicht allen Lernenden im FU } \\
\text { mitzuarbeiten, unabhängig von ihrem } \\
\text { Sprachniveau. }\end{array}$ & $\begin{array}{c}1 \\
3 \%\end{array}$ & $\begin{array}{c}2 \\
6 \%\end{array}$ & $\begin{array}{c}6 \\
18 \%\end{array}$ & $\begin{array}{c}7 \\
20 \%\end{array}$ & $\begin{array}{c}18 \\
53 \%\end{array}$ & $\begin{array}{c}34 \\
100 \%\end{array}$ & 4,15 & 1,09 \\
\hline 18. DP im FU fördert kooperatives Lernen. & $\begin{array}{c}0 \\
0 \%\end{array}$ & $\begin{array}{c}1 \\
3 \%\end{array}$ & $\begin{array}{c}9 \\
27 \%\end{array}$ & $\begin{array}{c}8 \\
24 \%\end{array}$ & $\begin{array}{c}16 \\
47 \%\end{array}$ & $\begin{array}{c}34 \\
100 \%\end{array}$ & 4,15 & 0,91 \\
\hline $\begin{array}{l}\text { 19. DP im FU ermöglicht ein aktives } \\
\text { Fremdsprachenlernen. }\end{array}$ & $\begin{array}{c}1 \\
3 \%\end{array}$ & $\begin{array}{c}0 \\
0 \%\end{array}$ & $\begin{array}{c}7 \\
20 \%\end{array}$ & $\begin{array}{c}11 \\
32 \%\end{array}$ & $\begin{array}{c}15 \\
45 \%\end{array}$ & $\begin{array}{c}34 \\
100 \%\end{array}$ & 4,15 & 0,94 \\
\hline $\begin{array}{l}\text { 20. DP ermöglicht Lernenden die } \\
\text { Zielsprache auf eine kommunikative } \\
\text { Weise zu lernen. }\end{array}$ & $\begin{array}{c}0 \\
0 \%\end{array}$ & $\begin{array}{c}1 \\
3 \%\end{array}$ & $\begin{array}{c}4 \\
12 \%\end{array}$ & $\begin{array}{c}7 \\
20 \%\end{array}$ & $\begin{array}{c}22 \\
65 \%\end{array}$ & $\begin{array}{c}34 \\
100 \%\end{array}$ & 4,47 & 0,81 \\
\hline $\begin{array}{l}\text { 21. Mit DP kann die Individualität der } \\
\text { Lernenden im FU besser zum Ausdruck } \\
\text { gebracht werden. }\end{array}$ & $\begin{array}{c}0 \\
0 \%\end{array}$ & $\begin{array}{c}1 \\
3 \%\end{array}$ & $\begin{array}{c}8 \\
24 \%\end{array}$ & $\begin{array}{c}11 \\
32 \%\end{array}$ & $\begin{array}{c}14 \\
41 \%\end{array}$ & $\begin{array}{c}34 \\
100 \%\end{array}$ & 4,12 & 0,87 \\
\hline $\begin{array}{l}\text { 22. DP im FU ermöglicht einen } \\
\text { authentischen Sprachgebrauch. }\end{array}$ & $\begin{array}{c}0 \\
0 \%\end{array}$ & $\begin{array}{c}3 \\
9 \%\end{array}$ & $\begin{array}{c}9 \\
27 \%\end{array}$ & $\begin{array}{c}13 \\
38 \%\end{array}$ & $\begin{array}{c}9 \\
27 \%\end{array}$ & $\begin{array}{c}34 \\
100 \%\end{array}$ & 3,82 & 0,92 \\
\hline $\begin{array}{l}\text { 23. Mit DP wird die Fremdsprache in einem } \\
\text { realen Kontext gelernt. }\end{array}$ & $\begin{array}{c}0 \\
0 \% \\
\end{array}$ & $\begin{array}{c}3 \\
9 \% \\
\end{array}$ & $\begin{array}{c}7 \\
20 \% \\
\end{array}$ & $\begin{array}{c}11 \\
32 \% \\
\end{array}$ & $\begin{array}{c}13 \\
38 \% \\
\end{array}$ & $\begin{array}{c}34 \\
100 \%\end{array}$ & 4,00 & 0,97 \\
\hline $\begin{array}{l}\text { 24. DP im FU unterstützt } \\
\text { Problemlösungen. }\end{array}$ & $\begin{array}{c}0 \\
0 \%\end{array}$ & $\begin{array}{c}7 \\
20 \%\end{array}$ & $\begin{array}{c}14 \\
41 \%\end{array}$ & $\begin{array}{c}6 \\
18 \%\end{array}$ & $\begin{array}{c}7 \\
20 \%\end{array}$ & $\begin{array}{c}34 \\
100 \%\end{array}$ & 3,38 & 1,03 \\
\hline $\begin{array}{l}\text { 25. DP im FU eignet sich nur zur } \\
\text { gelegentlichen Belebung des } \\
\text { Unterrichtsstoffes. }\end{array}$ & $\begin{array}{c}1 \\
3 \%\end{array}$ & $\begin{array}{c}7 \\
20 \%\end{array}$ & $\begin{array}{c}15 \\
45 \%\end{array}$ & $\begin{array}{c}7 \\
20 \%\end{array}$ & $\begin{array}{c}4 \\
12 \%\end{array}$ & $\begin{array}{c}34 \\
100 \%\end{array}$ & 3,18 & 0,98 \\
\hline
\end{tabular}

\subsubsection{Kategorie Emotionen}

Die Kategorie Emotionen bezieht sich auf die Gefühle der Lernenden, die das Lernen mithilfe der Dramapädagogik einerseits fördern, andererseits gewisse Hemmungen verursachen oder das Lernen ver- bzw. behindern. Aus den Ergebnissen in der Tabelle 8 kann zusammengefasst werden, dass sich dramapädagogische Elemente positiv auf die Stressreduktion auswirken, sowohl beim Sprechen $(\overline{\mathrm{x}}=4,32)$ wie auch im DaF-Unterricht 
im Allgemeinen $(\bar{x}=4,06)$. Eine positive Wirkung könnte sich auch in einer erhöhten Motivation für das Fremdsprachenlernen $(\overline{\mathrm{x}}=4,00)$ zeigen. Dennoch darf nicht übersehen werden, dass diese Methode nicht allen Lernenden auf den Leib geschrieben ist und gerade diese schauspielerischen Elemente im Unterricht für manche Teilnehmer Stress bedeuten können $(\overline{\mathrm{x}}=2,94)$. Eine ausführliche Einleitung in die dramapädagogische Herangehensweise und eine genaue Erklärung der Ziele ist unseres Erachtens von großer Bedeutung, damit die Lernenden den Sinn dieser Methode einsehen und eventuell negative Emotionen abbauen. Der Mittelwert für die Kategorie Emotionen ist 3,83 und damit ist Dramapädagogik als eine Herangehensweise mit mittelmäßiger Effizienz positioniert.

Tabelle 8 Emotionen

\begin{tabular}{|c|c|c|c|c|c|c|c|c|}
\hline \multirow[t]{2}{*}{ Frage 6 - Aussagen } & 1 & 2 & 3 & 4 & 5 & \multirow[t]{2}{*}{$\mathbf{N}$} & \multirow[t]{2}{*}{$(\mathbf{x})$} & \multirow{2}{*}{$\begin{array}{l}(\sigma) \\
\text { Std. }\end{array}$} \\
\hline & \multicolumn{5}{|c|}{ Grad der Zustimmung (f, fo) } & & & \\
\hline $\begin{array}{l}\text { 26. Mit DP kann besonders der Stress } \\
\text { beim Sprechen reduziert werden. }\end{array}$ & $\begin{array}{c}0 \\
0 \%\end{array}$ & $\begin{array}{c}1 \\
3 \%\end{array}$ & $\begin{array}{c}3 \\
9 \%\end{array}$ & $\begin{array}{c}14 \\
41 \%\end{array}$ & $\begin{array}{c}16 \\
47 \%\end{array}$ & $\begin{array}{c}34 \\
100 \%\end{array}$ & 4,32 & 0,76 \\
\hline $\begin{array}{l}\text { 27. DP senkt den Stresspegel im DaF- } \\
\text { Unterricht. }\end{array}$ & $\begin{array}{c}0 \\
0 \%\end{array}$ & $\begin{array}{c}2 \\
6 \%\end{array}$ & $\begin{array}{c}6 \\
18 \%\end{array}$ & $\begin{array}{c}14 \\
41 \%\end{array}$ & $\begin{array}{c}12 \\
35 \%\end{array}$ & $\begin{array}{c}34 \\
100 \%\end{array}$ & 4,06 & 0,87 \\
\hline $\begin{array}{l}\text { 28. DP wirkt sich positiv auf die Motivation } \\
\text { für das Fremdsprachenlernen aus. }\end{array}$ & $\begin{array}{c}0 \\
0 \%\end{array}$ & $\begin{array}{c}2 \\
6 \%\end{array}$ & $\begin{array}{c}9 \\
26 \%\end{array}$ & $\begin{array}{c}10 \\
29 \%\end{array}$ & $\begin{array}{c}13 \\
39 \%\end{array}$ & $\begin{array}{c}34 \\
100 \%\end{array}$ & 4,00 & 0,94 \\
\hline 29. DP fördert positive Emotionen. & $\begin{array}{c}0 \\
0 \%\end{array}$ & $\begin{array}{c}4 \\
12 \%\end{array}$ & $\begin{array}{c}6 \\
18 \%\end{array}$ & $\begin{array}{c}14 \\
41 \%\end{array}$ & $\begin{array}{c}10 \\
29 \%\end{array}$ & $\begin{array}{c}34 \\
100 \%\end{array}$ & 3,88 & 0,96 \\
\hline $\begin{array}{l}\text { 30. DP hilft Lernenden Empathie zu } \\
\text { entwickeln. }\end{array}$ & $\begin{array}{c}0 \\
0 \%\end{array}$ & $\begin{array}{c}4 \\
12 \%\end{array}$ & $\begin{array}{c}8 \\
24 \%\end{array}$ & $\begin{array}{c}14 \\
41 \%\end{array}$ & $\begin{array}{c}8 \\
24 \%\end{array}$ & $\begin{array}{c}34 \\
100 \%\end{array}$ & 3,76 & 0,94 \\
\hline $\begin{array}{l}\text { 31. DP verursacht Stress, weil Lernende } \\
\text { nicht gerne Theater spielen. }\end{array}$ & $\begin{array}{c}3 \\
9 \%\end{array}$ & $\begin{array}{c}5 \\
15 \%\end{array}$ & $\begin{array}{c}17 \\
50 \%\end{array}$ & $\begin{array}{c}9 \\
26 \%\end{array}$ & $\begin{array}{c}0 \\
0 \%\end{array}$ & $\begin{array}{c}34 \\
100 \%\end{array}$ & 2,94 & 0,87 \\
\hline
\end{tabular}

\subsubsection{Kategorie Entwicklung literarischer Kompetenzen}

Dramapädagogische Elemente sind eng mit dem Spielen im Unterricht verbunden und könnten den Eindruck vermitteln, dass diese Lehr-Lernmethode nicht für einen ernsthaften Umgang mit literarischen Texten geeignet sei. Viele Studierende äußerten nämlich vor dem Workshop Zweifel an der Effizienz dieser Methode im Literaturunterricht, denn es werde ja nur viel gespielt und nicht ernst gearbeitet. Ein Literaturunterricht sei aber keine Spielerei. Die Resultate der Umfrage für die Kategorie Entwicklung literarischer Kompetenzen zeigen ein anderes Bild. Die Mehrheit der Studierenden scheint nach der eigenen Erprobung der Methode davon überzeugt zu sein, dass Dramapädagogik auch für eine vertiefte Arbeit mit literarischen Texten eingesetzt werden kann und den Lernenden gleichzeitig Hilfe beim Verstehen leisten kann. Mit dem Mittelwert 4,37 wurde der dramapädagogischen Methode letztendlich eine hohe Effizienz für die Entwicklung literarischer Kompetenzen zugeschrieben. 
Tabelle 9 Literarische Kompetenzen

\begin{tabular}{|c|c|c|c|c|c|c|c|c|}
\hline \multirow[t]{2}{*}{ Frage 6 - Aussagen } & 1 & 2 & 3 & 4 & 5 & \multirow[t]{2}{*}{$\mathbf{N}$} & \multirow[t]{2}{*}{$(x)$} & \multirow{2}{*}{$\begin{array}{l}(\sigma) \\
\text { Std. }\end{array}$} \\
\hline & \multicolumn{5}{|c|}{ Grad der Zustimmung (f, f\%) } & & & \\
\hline $\begin{array}{l}\text { 32. Dramapädagogische Elemente im FU } \\
\text { sind auch für den anspruchsvollen } \\
\text { Literaturunterricht geeignet. }\end{array}$ & $\begin{array}{c}0 \\
0 \%\end{array}$ & $\begin{array}{c}1 \\
3 \%\end{array}$ & $\begin{array}{c}2 \\
6 \%\end{array}$ & $\begin{array}{c}4 \\
12 \%\end{array}$ & $\begin{array}{c}27 \\
79 \%\end{array}$ & $\begin{array}{c}34 \\
100 \%\end{array}$ & 4,67 & 0,72 \\
\hline $\begin{array}{l}\text { 33. Theaterinszenierungen im FU helfen } \\
\text { Lernenden ein literarisches Werk besser } \\
\text { zu verstehen. }\end{array}$ & $\begin{array}{c}1 \\
3 \%\end{array}$ & $\begin{array}{l}1 \\
3 \%\end{array}$ & $\begin{array}{c}8 \\
24 \%\end{array}$ & $\begin{array}{c}9 \\
26 \%\end{array}$ & $\begin{array}{c}15 \\
44 \%\end{array}$ & $\begin{array}{c}34 \\
100 \%\end{array}$ & 4,06 & 1,03 \\
\hline
\end{tabular}

\subsubsection{Kategorie Diverse Kompetenzen}

Der letzte Bereich, der in der Umfrage abgefragt wurde, war der Einfluss der Dramapädagogik auf die Entwicklung unterschiedlicher Kompetenzen, die sich nicht unbedingt auf den Fremdsprachenunterricht beziehen. Die Mehrheit der Studierenden glaubt, Dramapädagogik habe eine sehr positive Wirkung auf die Kreativität der Lernenden $(\overline{\mathrm{x}}=4,62)$, auf ein positives Selbstbild $(\overline{\mathrm{x}}=4,38)$, auf die Förderung der sozialen Kompetenz $(\bar{x}=4,21)$ und der Rolle der Körpersprache $(\bar{x}=4,15)$. Auch fächerübergreifendes Lernen, Interkulturalität und zwischenmenschliche Beziehungen wie in der Tabelle 10 aufgelistet, können laut den Studierenden mithilfe der Dramapädagogik recht positiv beeinflusst werden. Anhand der Resultate lässt sich feststellen, dass eine dramapädagogische Herangehensweise eine breite Palette an Kompetenzen anspricht, darunter auch diejenigen, die im Fremdsprachenunterricht nicht unbedingt im Vordergrund stehen, wie z. B. Körpersprache oder zwischenmenschliche Beziehungen. Die Dramapädagogik wird als effektive Methode für die Entwicklung diverser Kompetenzen bewertet $(\overline{\mathrm{x}}=4,07)$.

Tabelle 10 Diverse Kompetenzen

\begin{tabular}{|c|c|c|c|c|c|c|c|c|}
\hline \multirow[t]{2}{*}{ Frage 6 - Aussagen } & 1 & 2 & 3 & 4 & 5 & \multirow[t]{2}{*}{$\mathbf{N}$} & \multirow[t]{2}{*}{$\left(x^{-1}\right)$} & \multirow{2}{*}{$\begin{array}{l}(\sigma) \\
\text { Std. }\end{array}$} \\
\hline & \multicolumn{5}{|c|}{$\begin{array}{l}\text { Grad der Zustimmung } \\
(\mathrm{f}, \mathrm{f} \%)\end{array}$} & & & \\
\hline $\begin{array}{l}\text { 34. DP fördert die Kreativität der } \\
\text { Lernenden. }\end{array}$ & $\begin{array}{c}0 \\
0 \%\end{array}$ & $\begin{array}{c}0 \\
0 \%\end{array}$ & $\begin{array}{c}2 \\
6 \%\end{array}$ & $\begin{array}{c}9 \\
27 \%\end{array}$ & $\begin{array}{c}23 \\
67 \%\end{array}$ & $\begin{array}{c}34 \\
100 \%\end{array}$ & 4,62 & 0,59 \\
\hline $\begin{array}{l}\text { 35. DP hat einen positiven Einfluss auf das } \\
\text { Selbstbild der Lernenden. }\end{array}$ & $\begin{array}{c}0 \\
0 \%\end{array}$ & $\begin{array}{c}0 \\
0 \% \\
\end{array}$ & $\begin{array}{c}5 \\
15 \%\end{array}$ & $\begin{array}{c}11 \\
32 \%\end{array}$ & $\begin{array}{c}18 \\
53 \% \\
\end{array}$ & $\begin{array}{c}34 \\
100 \%\end{array}$ & 4,38 & 0,73 \\
\hline $\begin{array}{l}\text { 36. DP fördert die soziale Kompetenz der } \\
\text { Lernenden. }\end{array}$ & $\begin{array}{c}0 \\
0 \%\end{array}$ & $\begin{array}{c}1 \\
3 \%\end{array}$ & $\begin{array}{c}6 \\
18 \%\end{array}$ & $\begin{array}{c}12 \\
35 \%\end{array}$ & $\begin{array}{c}15 \\
44 \%\end{array}$ & $\begin{array}{c}34 \\
100 \%\end{array}$ & 4,21 & 0,83 \\
\hline $\begin{array}{l}\text { 37. Es ist wichtig, dass im FU auch } \\
\text { Körpersprache eine Rolle spielt . }\end{array}$ & $\begin{array}{c}0 \\
0 \%\end{array}$ & $\begin{array}{c}0 \\
0 \%\end{array}$ & $\begin{array}{c}8 \\
24 \%\end{array}$ & $\begin{array}{c}13 \\
38 \%\end{array}$ & $\begin{array}{c}13 \\
38 \%\end{array}$ & $\begin{array}{c}34 \\
100 \%\end{array}$ & 4,15 & 0,77 \\
\hline $\begin{array}{l}\text { 38. DP ist für das fächerübergreifende } \\
\text { Lernen geeignet. }\end{array}$ & $\begin{array}{c}0 \\
0 \%\end{array}$ & $\begin{array}{c}2 \\
6 \%\end{array}$ & $\begin{array}{c}10 \\
29 \%\end{array}$ & $\begin{array}{c}17 \\
50 \%\end{array}$ & $\begin{array}{c}5 \\
15 \%\end{array}$ & $\begin{array}{c}34 \\
100 \%\end{array}$ & 3,74 & 0,78 \\
\hline
\end{tabular}




\begin{tabular}{|c|c|c|c|c|c|c|c|c|}
\hline \multirow[t]{2}{*}{ Frage 6 - Aussagen } & 1 & 2 & 3 & 4 & 5 & \multirow[t]{2}{*}{$\mathbf{N}$} & \multirow[t]{2}{*}{$(\mathbf{x})$} & \multirow{2}{*}{$\begin{array}{l}(\sigma) \\
\text { Std. }\end{array}$} \\
\hline & \multicolumn{5}{|c|}{$\begin{array}{l}\text { Grad der Zustimmung } \\
(\mathrm{f}, \mathrm{f} \%)\end{array}$} & & & \\
\hline 39. DP im FU fördert die Interkulturalität. & $\begin{array}{c}0 \\
0 \%\end{array}$ & $\begin{array}{c}0 \\
0 \%\end{array}$ & $\begin{array}{c}15 \\
44 \%\end{array}$ & $\begin{array}{c}13 \\
38 \%\end{array}$ & $\begin{array}{c}6 \\
18 \%\end{array}$ & $\begin{array}{c}34 \\
100 \%\end{array}$ & 3,74 & 0,74 \\
\hline $\begin{array}{l}\text { 40. DP im FU fördert ein besseres fördert } \\
\text { ein besseres soziales Miteinander. }\end{array}$ & $\begin{array}{c}0 \\
0 \%\end{array}$ & $\begin{array}{c}4 \\
12 \%\end{array}$ & $\begin{array}{c}11 \\
32 \%\end{array}$ & $\begin{array}{c}11 \\
32 \%\end{array}$ & $\begin{array}{c}8 \\
24 \%\end{array}$ & $\begin{array}{c}34 \\
100 \%\end{array}$ & 3,68 & 0,96 \\
\hline
\end{tabular}

Zusammenfassend ist festzustellen, dass die angehenden DaF-Lehrenden in unserer Untersuchung eine positive Einstellung gegenüber Dramapädagogik haben bzw. entwickelt haben. Es besteht zwar keine Garantie, dass sich die zukünftigen DaF-Lehrenden später im Beruf dramapädagogischer Unterrichtselemente bedienen, dennoch gehen wir davon aus, dass sie aufgrund ihrer positiven Erfahrungen mit dieser Methode diese bei der Unterrichtsplanung zumindest in Betracht ziehen. Die Resultate der Umfrage deuten darauf hin, dass Studierende allgemein dem dramapädagogischen Ansatz in unterschiedlichen Bereichen des Fremdsprachenlernens eine bedeutende Effizienz zuschreiben. Eine hohe Effizienz dieser Methode ist laut der angehenden DaF-Lehrenden beim Vokabellernen zu verzeichnen, ebenso bei der Entwicklung von fremdsprachlicher Kompetenz, der Entwicklung literarischer Kompetenzen und der Entwicklung diverser Kompetenzen, die im Fremdsprachenunterricht gefördert werden können, aber oft im Hintergrund bleiben. Eine mittelmäßige Effizienz wurde für die Kategorien Grammatik, Lernprozess und Lernformen und Emotionen berechnet.

Die Verzahnung von Theorie und Praxis im Rahmen der Veranstaltung Pädagogisches Praktikum, die als ein dramapädagogischer Workshop stattfand, beurteilen wir aus folgenden Gründen als sehr gelungen:

- Die Studierenden äußerten zu Beginn des Workshops ihre eigene Meinung und versprachlichten bzw. offenbarten ihre subjektiven Theorien über die Effizienz dieser Methode, was sie dazu anregte, Argumente zu suchen, um ihre Meinung auch begründen zu können. Schon bei diesem Schritt konnten viele feststellen, dass sie eher auf eigenen Überzeugungen beharren und wenige oder keine Argumente haben. Durch die Versprachlichung ihrer Gedanken bzw. Beharrungen war vielen bewusst, dass sie sich beim Gewinnen neuer Erkenntnisse selbst im Wege stehen. Eine Verbindung der theoretischen Hintergründe mit Videosequenzen, die praktische Beispiele aus dem Unterricht zeigten, ermöglichte den Studierenden einzusehen, dass es um Theorie geht, die auch praktisch im Klassenzimmer leben kann und im Rahmen des Fremdsprachenunterrichts realisierbar ist.

- Die Erprobung einzelner dramapädagogischer Techniken erwies sich als äußerst nützlich, denn die Studierenden mussten sich in die jeweiligen Rollen hineinversetzen, in 
die Als-ob-Situationen eintauchen und selbst aktiv werden. Sie konnten anhand eigener Emotionen und Erfahrungen und anhand der Erfahrungen der anderen Beteiligten über einzelne Techniken reflektieren und dazu argumentiert Stellung nehmen.

Von diesen Erkenntnissen ausgehend, fassen wir zusammen, dass eine gut durchdachte Verzahnung von Theorie und Praxis schon während der Ausbildungszeit in vielerlei Hinsichten gewinnbringend sein kann. Einerseits kann dadurch das Verstehen unterschiedlicher pädagogischer Konzepte gestärkt werden und andererseits wird den Studierenden ermöglicht, sich mit eigenen subjektiven Theorien auseinanderzusetzen und möglicherweise ihre bisherigen Grenzen zu erweitern bzw. zu überschreiten. Die dramapädagogische Methode, die in den Anfangsgesprächen eher als spielerische und weniger effektive Herangehensweise bewertet worden war, erstrahlte so in einem ganz anderen Licht.

\section{LITERATUR}

BIRNBAUM, Theresa (2013) Die Rolle von kooperativem Lernen und Dramapädagogik in Bezug auf das fremdsprachliche Handeln - Aktionsforschung zum DaF-Theaterprojekt Entre bastidores mit den Physikern an der Universidad de Salamanca. Scenario Journal 7/ 1, 40-59.

BONNET, Andreas/Almut KÜPPERS (2011) Wozu taugen kooperatives Lernen und Dramapädagogik? Vergleich zweier populärer Inszenierungsformen. A. Küppers, T. Schmidt, M. Walter (Hg), Inszenierungen im Fremdsprachenunterricht. Grundlagen, Formen, Perspektiven. Hannover: Schroedel, 32-51.

BÜNGER, Ursula Christine (2010) Individualisierung und Fiktionalisierung der KannBeschreibungen des Europäischen Referenzrahmens durch Dramapädagogik mit Handyfilmen. Scenario Journal 2, 42-62.

DRAMAPÄDAGOGIK Sprachen lehren und lernen mit Drama und Theater, https://dramapaedagogik.de/de/, 27. Juli 2020.

EVEN, Susanne/ Manfred SCHEWE (Hg.) (2016) Performatives Lehren, Lernen, Forschen - Performative Teaching, Learning, Research. Berlin: Schibri.

HAACK, Adrian (2018) Dramapädagogik, Selbstkompetenz und Professionalisierung. Performative Identitätsarbeit im Lehramtsstudium Englisch. Wiesbaden: Metzler/Springer. JOHNSTONE, Keith (2010) Improvisation und Theater. Berlin: Alexander Verlag. KIRSCH, Dieter (2013) Szenisches Lernen. Theaterarbeit im DaF-Unterricht. Ismaning: Hueber Verlag.

HAFTNER, Magdalena/Anne-Marie KUHFUSS (2014) Ich habe gar nicht gemerkt, dass ich Deutsch spreche - Wie die theatrale Erfahrung des Improvisationstheaters freies und authentisches Sprechen erlebbar macht und die kommunikative Kompetenz 
fördert. N. Bernstein, C. Lerchner (Hg.), Ästhetisches Lernen im DaF-/DaZ-Unterricht. Literatur - Theater - Bildende Kunst-Musik-Film. Göttingen: Universitätsverlag Göttingen, 217-233.

KALIN, Jana (2004) »Izkušnja - refleksija - delovanje« v procesu profesionalnega razvoja učitelja. B. Marentič Požarnik (ur.), Konstruktivizem v šoli in izobraževanje učiteljev. Ljubljana: Center za pedagoško izobraževanje Filozofske fakultete, 597-611.

KESSLER, Benedikt (2008) Interkulturelle Dramapädagogik. Dramatische Arbeit als Vehikel des interkulturellen Lernens im Fremdsprachenunterricht. Frankfurt am Main.: Lang.

KOSEVSKI PULJIĆ, Brigita/Andreja RETELJ (2019) Poti in stranpoti nemščine na Slovenskem. T. BALAŽIC BULC et. al. (ur.), Poti in stranpoti poučevanja tujih jezikov v Sloveniji. 1. izd. Ljubljana: Znanstvena založba Filozofske fakultete, 175-186.

NEUMAYER Ulrike (2004) Eine (Sprach)Reise. Dramapädagogik im Fremdsprachenunterricht. Ein ganzheitlicher Lehr- und Lernansatz für GermanistikstudentInnen in Spanien? M. J. Domingues, B. Lübke, A. Mallo (Hg), El alemán en su contexto espanol / Deutsch im spanischen Kontext, Actas IV Congreso (4. Germanisten und Deutschlehrerkongress) de la Federacion de Asociaciones de Germanistas y Profesores de Alemán en Espana (FAGE) 2002, Santiago de Compostela: Publicacions, 503-514.

SAMBANIS, Michaela (2013) Fremdsprachenunterricht und Neurowissenschaften. Tübingen: Narr Francke.

SAMBANIS, Michaela (2016) Dramapädagogik im Fremdsprachenunterricht - Überlegungen aus didaktischer und neurowissenschaftlicher Sicht. S. Even, M. Schewe (Hg.), Performatives Lehren, Lernen, Forschen - Performative Teaching, Learning, Research. Berlin: Schibri, S. 47-66.

SCHEWE, Manfred (1993) Fremdsprache inszenieren : zur Fundierung einer dramapädagogischen Lehr- und Lernpraxis. Oldenburg: Zentrum für pädagogische Berufspraxis.

SCHMENK, Barbara (2004) Drama in the Margins? The Common European Framework of Reference and its Implications for Drama Pedagogy in the Foreign Language Classroom. German as a Foreign Language (GFL-Journal) 1, 7-23. 15. Juli 2020. http://www.gfl-journal.de/1-2004/schmenk.pdf.

TSELIKAS, Elektra I (1999) Dramapädagogik im Sprachunterricht. Zürich: Orell Füssli. WITTAL-DÜERKOP, Tanya. (2019) Lehren, Lernen, Handeln - Potentiale und Anwendungsfelder der Dramapädagogik im Sprachunterricht. S. Giebert, E. Göksel (Hg.), Dramapädagogik-Tage 2018/Drama in Education Days 2018 - Conference Proceedings of the 4th Annual Conference on Performative Language Teaching and Learning. 12-22. 15. Juli 2020 https://dramapaedagogik.de/wp-content/uploads/ proceedings2018/final.pdf. 


\section{POVZETEK}

\section{MNENJA BODOČIH UČITELJEV NEMŠČINE O UČINKOVITOSTI DRAMSKE PEDA- GOGIKE ZA UČENJE TUJIH JEZIKOV}

Prispevek obravnava metodo dramske pedagogike z vidika učinkov na poučevanje in učenje tujih jezikov. Uvodoma obravnavamo razlikovanje med metodama dramska in gledališka pedagogika, ki se je uveljavilo na področju tujejezikovne didaktike in nato predstavimo poglavitne značilnosti dramske pedagogike. Osrednji del članka predstavlja rezultate ankete med bodočimi učitelji/učiteljicami nemščine o učinkovitosti metode dramska pedagogika za razvijanje različnih zmožnosti pri pouku tujega jezika. Iz analize rezultatov izhaja, da bodoči učitelji in učiteljice nemščine prepoznavajo največje prednosti te metode pri razvijanju literarnih kompetenc, učenju besedišča, splošni tujejezikovni zmožnosti in tudi razvijanju različnih kompetenc, ki so pri pouku tujega jezika velikokrat v ozadju. Srednje učinke ima metoda dramske pedagogike po mnenju študentov na sam učni proces oziroma samoregulacijo učenja in učne oblike, na razvijanje slovnice in na emocije pri pouku. Negativnih učinkov na poučevanje ali učenje tujega jezika s pomočjo dramske pedagogike študenti ne zaznavajo. Delavniška oblika spoznavanja elementov dramske pedagogike se je izkazala kot zelo uspešna oblika povezovanja teorije in prakse v času študija, saj je študentom olajšala osmišljanje teorije, omogočila usvojitev praktičnih veščin in jih spodbudila k refleksiji tako lastnega učenja kot tudi obravnavane učne metode. Poleg pridobivanja osebnih izkušenj in praktičnih veščin se takšna reflektivno usmerjena oblika učenja izkaže kot zelo učinkovita za razvijanje kompetence samorefleksije, ki je ena od ključnih učiteljskih kompetenc.

Ključne besede: dramska pedagogika, učne metode, učne tehnike, izobraževanje bodočih učiteljev, delavnica, nemščina kot tuji jezik

\section{ABSTRACT}

\section{PRE-SERVICE TEACHERS' ATTITUDES ON THE EFFICIENCY OF DRAMA EDUCA- TION IN LEARNING AND TEACHING FOREIGN LANGUAGES}

The aim of this article is to introduce drama education as a method of learning and teaching foreign languages. We first introduce the distinction between two methods: drama education and theatre pedagogy, as established in the field of foreign language didactics, and then present some main characteristics of the method of drama education.

The main part of the article is based on the results of a survey among pre-service teachers of German as a foreign language about the efficiency of drama education for the development of various competences in the foreign language classroom. The analysis shows the greatest benefit of drama education for the development of literary competences, vocabulary learning, foreign 
language skills and other competences, that are not so frequently developed in foreign language teaching. The average impact of drama education in this context as assessed with regard to emotions, development of grammar competence, learning process and learning styles. The students did not recognise any negative effects of drama education on foreign language teaching or learning. Workshops on drama education seem to be a very successful way of combining theory and practice in pre-service teacher training. It helped students to better understand the theory, gain practical knowledge and reflect on their own learning as well as the drama-based pedagogical method. Besides gaining personal experience and practical techniques, providing a workshop with a focus on reflection a very effective way to develop the competence of self-reflection, which is one of the key competences of teachers.

Keywords: drama education, drama pedagogy, learning methods, learning techniques, pre-service teacher education, German as a foreign language 